\title{
A unified neurocomputational bilateral model of spoken language production in healthy participants and recovery in poststroke aphasia
}

\author{
Ya-Ning Chang ${ }^{\mathrm{a}, 1}$ (1) and Matthew A. Lambon Ralph ${ }^{\mathrm{a}, 1}$ \\ ${ }^{a}$ Medical Research Council Cognition and Brain Sciences Unit, University of Cambridge, Cambridge CB2 7EF, United Kingdom
}

Edited by Cathy J. Price, University College London, London, United Kingdom, and accepted by Editorial Board Member Leslie G. Ungerleider November 2, 2020 (received for review May 21, 2020)

\begin{abstract}
Understanding the processes underlying normal, impaired, and recovered language performance has been a long-standing goal for cognitive and clinical neuroscience. Many verbally described hypotheses about language lateralization and recovery have been generated. However, they have not been considered within a single, unified, and implemented computational framework, and the literatures on healthy participants and patients are largely separated. These investigations also span different types of data, including behavioral results and functional MRI brain activations, which augment the challenge for any unified theory. Consequently, many key issues, apparent contradictions, and puzzles remain to be solved. We developed a neurocomputational, bilateral pathway model of spoken language production, designed to provide a unified framework to simulate different types of data from healthy participants and aphasic patients. The model encapsulates key computational principles (differential computational capacity, emergent division of labor across pathways, experience-dependent plasticityrelated recovery) and provides an explanation for the bilateral yet asymmetric lateralization of language in healthy participants, chronic aphasia after left rather than right hemisphere lesions, and the basis of partial recovery in patients. The model provides a formal basis for understanding the relationship between behavioral performance and brain activation. The unified model is consistent with the degeneracy and variable neurodisplacement theories of language recovery, and adds computational insights to these hypotheses regarding the neural machinery underlying language processing and plasticityrelated recovery following damage.
\end{abstract}

stroke aphasia | language lateralization | language recovery | bilateral language processing | neurocomputational modeling

anguage is a key human ability, and, when impaired (e.g., after - stroke or neurodegeneration), patients are left with significant disability. Aphasia (acquired language impairments that follow from brain injury, affecting comprehension, production, reading, and writing) is common (1). Studies of healthy and impaired language have a long history, and these vibrant literatures have generated many verbally described hypotheses. The long-standing literature on aphasia dates back to seminal 19th century studies (2-4). While these verbally described hypotheses advanced our understanding of language processing both theoretically and clinically, it is not clear how they work mechanistically, and they can be mutually incompatible. For instance, some notions propose good aphasia recovery only results from language returning to the left hemisphere (5-9), while others report that recovered language performance is positively correlated with activation in the right hemisphere (10-12). As a recent review (13) noted, the current situation is confusing because there are many individual findings and different types of data (e.g., patients' language performance vs. functional MRI [fMRI] activations), yet no unified mechanistic account. There is a pressing need to have an implemented neurocomputational model which can provide 1) a unified framework in which findings from healthy participants and aphasic patients can be accounted for, 2) a computationally instantiated framework to formalize and test verbally described hypotheses, and 3) a framework that can bridge between different types of cognitive neuroscience data including language behavior, lesion locations, and task-related fMRI. This was the overarching aim of the current study. The puzzles and targets are set out briefly below.

\section{Lateralization Assumptions}

The first issue concerns lateralization in healthy and impaired language. The very strongly held view that language is a left hemisphere function primarily arises from the long-standing neuropsychology literature showing that chronic aphasia is associated with left hemisphere damage but not with right hemisphere damage (14-16). However, the patient data are more graded than often portrayed. Recent evidence has shown that right hemisphere lesions can generate language problems, especially in the early phase, and some mild remaining deficits can be measured in chronic cases (17). Several patient studies of semantic cognition (18-20) also show that bilateral damage is required, to show more substantial deficits.

Additionally, functional neuroimaging in healthy participants shows that many language tasks such as repetition, picture naming, comprehension, and production might be bilaterally supported (21-26). Although the activation patterns are often leftward asymmetric, the degree of asymmetry largely depends on the nature

\section{Significance}

Studies of healthy and impaired language have generated many verbally described hypotheses. While these verbal descriptions have advanced our understanding of language processing, some explanations are mutually incompatible, and it is unclear how they work mechanistically. We constructed a neurocomputational bilateral model of spoken language production to simulate a range of phenomena in healthy participants and patients with aphasia simultaneously, including language lateralization, impaired performance after left but not right damage, and hemispheric involvement in plasticity-dependent recovery. The model demonstrates how seemly contradictory findings can be simulated within a single framework. This provides a coherent mechanistic account of language lateralization and recovery from poststroke aphasia.

Author contributions: Y.-N.C. and M.A.L.R. designed research, performed research, analyzed data, and wrote the paper.

The authors declare no competing interest.

This article is a PNAS Direct Submission. C.J.P. is a guest editor invited by the Editorial Board.

This open access article is distributed under Creative Commons Attribution License 4.0 (CC BY).

${ }^{1}$ To whom correspondence may be addressed. Email: Matt.Lambon-Ralph@mrc-cbu.cam. ac.uk or yaning.chang@mrc-cbu.cam.ac.uk.

This article contains supporting information online at https://www.pnas.org/lookup/suppl/ doi:10.1073/pnas.2010193117/-/DCSupplemental.

First published December 3, 2020. 
of the tasks, with a subset showing stronger forms of asymmetric bias. For instance, propositional speech production is more left lateralized, whereas nonpropositional speech (e.g., counting) generates bilateral activations (27-29). Of course, identifying activations associated with language does not necessarily imply that the regions are necessary for language functions (30). Thus, it is important to note that a number of transcranial magnetic stimulation (TMS) studies of semantics (31-34) and phonology (35) also indicate that left and right areas contribute to healthy language.

Thus, when considering both chronic aphasic patients and healthy participants, it appears difficult to reconcile the seemingly contradictory findings: How can the language network be strongly left lateralized in patients but be bilateral, albeit asymmetric, in healthy participants? We propose that these results could reflect the outcome of an intrinsically bilateral yet asymmetric language network. Indeed, it has been demonstrated that functional asymmetry could follow from hemispheric asymmetry in the healthy language system (36-42) and that, when the system is damaged (e.g., in patients with low-grade glioma), the degree of asymmetry can change through functional plasticity $(43,44)$. For instance, using a combination of fMRI and diffusion tensor imaging, Vernooij et al. (42) demonstrated a significant correlation between functional hemispheric lateralization and the relative asymmetry of the arcuate fasciculus in healthy participants (see also ref. 41). Thus, within the language network, the majority of healthy participants show leftward asymmetry of brain volumes and arcuate fasciculus $(36,40,45)$, suggesting that more of the computational capacity is in the left than the right hemisphere. Such capacity imbalance might generate bilateral yet asymmetric lateralization in fMRI activation and also greater likelihood of chronic impairment after left than right damage. The latter may reflect a combination of the premorbid division of labor for left over right in healthy language, and the potential for plasticity-related recovery. This was explored in past computational work by reexposing the damaged model to its learning environment, generating plasticity-related recovery via "retuning" of the remaining computational capacity $(46,47)$. A straightforward hypothesis, from these earlier models, is that the potential for such recovery reflects the amount of computational capacity available. Thus, if the right hemisphere has insufficient capacity to learn all language functions by itself (SI Appendix, Supplementary Simulations), then, when the dominant left hemisphere is entirely damaged, language functions cannot be fully reestablished by the right hemisphere alone-resulting in chronic aphasia. In contrast, if the weaker right hemisphere is lesioned, then the dominant left hemisphere may have sufficient spare capacity to assimilate the extra work.

\section{The Computational Bases of Language Recovery}

A recent review (13) considered two mechanisms: degeneracy and variable neurodisplacement. Although not mutually exclusive, degeneracy (30) suggests that cognitive functions might arise from multiple, structurally distinct neural networks resulting in a partially resilient system. Following damage, recovery of function could be achieved by up-regulation of quiescent regions, alternative pathways, or nonlanguage regions that are not typically engaged in the healthy state (for a computationally implemented example, see ref. 46). The second mechanism is variable neurodisplacement, a concept borrowed from automotive engineering (variable displacement; https://en.wikipedia.org/wiki/Variable displacement). Given that the brain is metabolically expensive, it seems very likely that energy consumption needs to be balanced against performance demand. This can be achieved in engines by "displacing" (down-regulating or turning off) a subset of cylinders when full power is not required. Returning to the brain, it is well established that higher neural activity is coupled with increased metabolic energy costs (compare to neurovascular coupling). If we assume that a cognitive function is supported by a dynamic distributed network, then, when performance demand is not maximal, parts of this network could be "displaced" (downregulated) to save energy $(31,48)$. This displaced "spare capacity" is used when performance demand is high, but it could also be permanently up-regulated, after partial damage to the network, to support recovered performance (returning to the engine analogy, if one cylinder's function was compromised, then the other cylinders' output could be up-regulated to compensate).

Previous computational studies $(46,47)$ of plasticity-related recovery have provided some support for these principles, and highlighted two types of experience-dependent learning, each depending on remaining capacity in the model. In single pathway models, relearning can retune and activate the "perilesional" units and weights. Secondly, if there are multiple routes that support the task, relearning can also shift the division of labor between different pathways. The potential for recovery-related changes is determined by the capacity available in different pathways and their engagement in the task prior to damage. Both mechanistic hypotheses about language recovery need to be specified in more detail within an implemented computational model that can simulate healthy and impaired language, as well as generate the different measures used to assess recovery of function, such as language performance and fMRI activations.

\section{Theories of Aphasia Recovery}

In the long-standing literature on language recovery, most hypotheses are verbally described or are verbal descriptions of observed phenomena (13). For example, up-regulated activation in perilesional and contralesional areas has been associated with recovered performance in poststroke aphasia $(5,49-54)$. Van Oers et al. (53), for instance, showed that recovery of picture naming was associated with activation in the remaining portion of the left inferior frontal gyrus (IFG)- while recovery on moredemanding tasks was associated with up-regulated contralesional activation in the right IFG in addition to the left IFG. There is also parallel evidence from combined TMS-fMRI studies in healthy participants that inhibition of left hemisphere regions up-regulates activation in the right homologous regions $(31,32)$

Another notion is the right hemisphere hypothesis ( $\mathrm{RHH}$ ). Several neuroimaging studies have demonstrated that patients with left hemisphere damage recruit the right hemisphere during language tasks $(8,50,55)$. These findings have been interpreted in terms of a right hemisphere juvenile language system, which can provide some function after significant left hemisphere damage but it is generally weaker and error prone. Despite being a commonly repeated hypothesis for over a century, the computational mechanisms involved in the development of language and the shifts of function after damage remain unspecified and computationally unimplemented. More confusingly, the hypotheses and data in relation to the RHH are contradictory. Some notions suggest that aphasia recovery is supported by this right hemisphere system: Language performance is correlated with activation in the right hemisphere (10-12), and, when aphasic patients have a second right hemisphere stroke, their language performance declines $(2,56)$. In contrast, the "regional hierarchy framework" proposes that right hemisphere activation is maladaptive, resulting from a release of transcallosal inhibition, and good recovery only results from language returning to the left (5-9). In a seminal study of very mildly aphasic patients with good recovery (50), left hemisphere activation for auditory comprehension greatly decreased a few days after stroke and was followed by increased bilateral activation with a significantly up-regulated peak in the right hemisphere two weeks after stoke, and then the peak activation shifted back to the left hemisphere in the chronic phase. However, it remains unclear what mechanisms underlie the changes in brain activity and what the longitudinal patterns are for moderate and severe aphasia. 
These contradictory RHHs have inspired neurostimulation interventions with opposite aims: either promoting right hemisphere engagement (57) or trying to suppress it in favor of left hemisphere involvement (58-61). Without a better understanding of underlying mechanisms and a formal implemented model, various foundational issues remain. These include how a right hemisphere system can develop if it is suppressed by the left hemisphere; how the two systems might interact; and whether the results of negative associations between right hemisphere activation and language are simply a reflection of severity, as mild aphasia is associated with small lesions which leaves more of the left hemisphere intact and able to be activated. Our working assumption is that there is an intrinsically bilateral, albeit asymmetrically provisioned single functional network. That is, the left and right hemispheres both contribute to speech production, with differential contributions arising from the effects of imbalanced capacity across the hemispheres. An implemented model would permit a proper investigation of how this division of labor might shift and under what conditions, after brain damage.

Additionally, the hypothesis of the maladaptive right hemisphere activation supposes that the two hemispheres attempt to inhibit each other through transcallosal inhibition $(6,7,9)$. There are several puzzles about this hypothesis, including 1) why the healthy brain might spend most of its lifetime preventing regions from working (a biologically expensive implementation) and 2) how the less dominant system can develop even semiuseful representations if being persistently suppressed. We also note that, to the best of our knowledge, outside of the motor system (62-64), there are no demonstrations of transcallosal inhibitory connectivity. Conversely, there is even some evidence of excitatory connectivity $(65,66)$. With an implemented bilateral language model, we can explore the effect of transcallosal connectivity on model behavior, task performance, and recovery.

\section{Multiple Measures}

The last issue concerns different types of data and measures. Classically, explorations of brain function relied on relating brain activations $(8,10-12,52)$ or lesions $(67-69)$ to patients' performance. Functional neuroimaging now allows the healthy and damaged brain to be explored, in vivo. Thus we now have multiple measures to consider in parallel, including lesion location and size, behavioral language performance, activations, and connectivity. To make progress, the field needs to understand the relationship between these measures. It is tempting to assume that activated regions must be contributing to patients performance, but activation does not prove necessity (30). Furthermore, different types of analyses, such as multivoxel pattern analysis (MVPA) (70), have started to be used to explore and predict recovered performance. For instance, Fischer-Baum et al. (71) reported that, in a stroke patient with a severe reading impairment, the orthographic activation patterns in the right fusiform gyrus were more similar to stimulus patterns than in the left fusiform gyrus. Thus, it is critical that computational models are designed to accommodate multiple measures within a single framework, to allow formal explorations of the relationship between brain activations and contributions to the observed behavioral performance.

To summarize, the primary aim of this study is to address four key issues by developing a unified, bilateral pathway model of spoken language production: 1) language lateralization in healthy participants and poststroke patients, 2) mechanistic accounts for language recovery, 3) dynamic shifts of activation in poststroke aphasia and recovery with/without transcallosal connectivity, and 4) the relationship between multiple measures and recovered function. We directly compared the model to data derived from four important, exemplar studies of healthy individuals and poststroke aphasia-Vernooij et al. (42), Gajardo-Vidal et al. (17),
Saur et al. (50) and Fischer-Baum et al. (71) — and used the model to make predictions for future exploration. Given the maelstrom of historical hypotheses, data, etc., we have provided a summary guide to the critical issues, alternative viewpoints, our working hypotheses, and simulated effects, in Table 1.

\section{Results}

Hemispheric Asymmetry and Language Lateralization. The bilateral model was implemented as a simple recurrent network, consisting of two parallel pathways trained to perform word repetition (see Methods). We investigated whether the model could simulate language lateralization that follows hemispheric asymmetry, similar to the correlation pattern between functional hemispheric asymmetry in parietotemporal regions and structural asymmetry in arcuate fasciculus during the spoken production task reported in Vernooij et al. (42). Specifically, we varied the proportion of hidden units in the left versus the right pathways in the model (Fig. $1 A$ ) to simulate the relative capacity of the two pathways (72) while the total number of hidden units remained unchanged. The number of units for the two consecutive hidden layers in each pathway was the same. After training, the model was also tested on nonword repetition (an assessment of generalization to novel phonological forms).

In imaging studies, a laterality index is commonly estimated using blood oxygenation level dependent (BOLD) or cerebral blood flow in the left and right homolog language areas $(42,73)$. In our model, two different measures were used to compute the degree of lateralization: functional contribution and output unit activation. Functional contribution measured the relative contribution from the left or right pathway to output activation, as a proxy of effective connectivity analyses $(47,74)$. Output unit activation measured average unit activation at the output layer from either the left or right pathway, as a proxy of fMRI activation (75). A positive laterality score indicated that the model showed a left-lateralized pattern; conversely, a negative score indicated a right-lateralized pattern. An asymmetry index for computational capacity (number of left vs. right hidden units) was calculated in the same way as the laterality index. We also investigated average hidden unit activation in the left and right pathways across different conditions, during development and in recovery.

Results are summarized in Fig. 1. All models performed well on word repetition and generalized to nonwords (Fig. $1 B$ ). There was a clear lexicality effect, with the highest accuracy for highfrequency (HF) words, followed by low-frequency (LF) words and then nonwords (NW). Importantly, the performance level achieved by the model with differential capacities in the left and right was very similar because the total number of units was the same. These observations were confirmed by a repeated ANOVA. There was a significant word type effect (HF vs. LF vs. NW: $F(2,278)=33.8, P<0.001)$, while both model type $(P=$ $0.44)$ and its interaction with word type $(P=0.18)$ were not significant. This means that the model was able to exploit the computational capacity flexibly to learn the task and to generalize. In contrast, the underlying processing changed. Fig. $1 C$ shows that more hidden units along a pathway resulted in higher average hidden unit activation. Thus, the emergent functional division of labor in the model was not solely based on there being more units in the "dominant" processing pathway, but they also resultantly worked harder, on average. Critically, Fig. $1 D$ shows that the model with more processing units in the left pathway (i.e., a larger asymmetry index) produced a more left-lateralized pattern. Laterality indices based on functional contribution and output unit activation were both positively correlated with asymmetry index for model capacity, Pearson's $r=0.929, P<$ 0.001 , and Pearson's $r=0.917, P<0.001$, respectively. The results are consistent with the function-structure pattern reported in Vernooij et al. (42). 
Table 1. Overview of critical issues, key issues, alternative viewpoints, working hypotheses, and simulated effects/model explorations

\begin{tabular}{|c|c|c|c|c|}
\hline Critical issues & Key issues & Alternative viewpoints & Working hypotheses & Simulated effects/explorations \\
\hline $\begin{array}{l}\text { Lateralization } \\
\text { assumptions }\end{array}$ & $\begin{array}{l}\text { How can the language } \\
\text { network be strongly left } \\
\text { lateralized in patients but } \\
\text { be bilateral, albeit } \\
\text { asymmetric, in healthy } \\
\text { participants? }\end{array}$ & $\begin{array}{l}\text { 1) Impaired language function } \\
\text { after left but not right } \\
\text { hemisphere damage (14-16) } \\
\text { 2) Bilateral and asymmetric } \\
\text { brain activations in healthy } \\
\text { individuals during language } \\
\text { tasks (21-29) }\end{array}$ & $\begin{array}{l}\text { Leftward hemispheric } \\
\text { asymmetry generates } \\
\text { bilateral yet asymmetric } \\
\text { lateralization in simulated } \\
\text { BOLD and also greater } \\
\text { likelihood of chronic } \\
\text { impairment after left than } \\
\text { right damage }\end{array}$ & $\begin{array}{l}\text { 1) Functional asymmetry } \\
\text { followed computational } \\
\text { capacity2) A function- } \\
\text { structure pattern (42)3) } \\
\text { Impaired performance after } \\
\text { left but not right damage to } \\
\text { the model (17) }\end{array}$ \\
\hline $\begin{array}{l}\text { The computational } \\
\text { bases of language } \\
\text { recovery }\end{array}$ & $\begin{array}{l}\text { A lack of an implemented } \\
\text { model for the } \\
\text { computational bases of } \\
\text { language recovery }\end{array}$ & $\begin{array}{l}\text { 1) Degeneracy }(13,30) \\
\text { 2) Variable neurodisplacement } \\
\text { (13) }\end{array}$ & $\begin{array}{l}\text { The mechanisms are not } \\
\text { mutually exclusive and they } \\
\text { can be utilized as a part of } \\
\text { the recovery process }\end{array}$ & $\begin{array}{l}\text { Various analyses on model } \\
\text { behavior and explorations } \\
\text { of the underpinning } \\
\text { computations in both } \\
\text { damage and undamaged } \\
\text { conditions }\end{array}$ \\
\hline $\begin{array}{l}\text { Theories of aphasia } \\
\text { recovery }\end{array}$ & $\begin{array}{l}\text { 1) What are the dynamic } \\
\text { activation shifts in } \\
\text { poststroke aphasia and } \\
\text { recovery? } \\
\text { 2) What is the effect of } \\
\text { transcallosal connectivity } \\
\text { on healthy and impaired } \\
\text { function? }\end{array}$ & $\begin{array}{l}\text { 1) Perilesional up-regulated } \\
\text { activation }(5,49-54) 2) \text { The } \\
\text { "regional hierarchy } \\
\text { framework" (5-9) } \\
\text { 3) Right hemisphere activation } \\
(2,8,10-12,50,55,56)\end{array}$ & $\begin{array}{l}\text { Patterns of recovery are } \\
\text { related to the differential } \\
\text { capacity available in left } \\
\text { and right hemisphere } \\
\text { systems and lesion severity }\end{array}$ & $\begin{array}{l}\text { 1) Dynamic patterns of } \\
\text { activation shifts during } \\
\text { recovery (50)2) Lesion severity } \\
\text { as a determiner for recovered } \\
\text { performance and brain } \\
\text { activation patterns3) Different } \\
\text { types of interconnectivity }\end{array}$ \\
\hline Multiple measures & $\begin{array}{l}\text { What is the relationship } \\
\text { between multiple brain } \\
\text { measures and recovered } \\
\text { function in patients? }\end{array}$ & $\begin{array}{l}\text { 1) Behavioral measures } \\
\text { compared with fMRI } \\
\text { activation }(8,10-12,52) \\
\text { 2) Potential applications of } \\
\text { multiple voxel pattern } \\
\text { analysis }(71,72) \text { in the } \\
\text { patient studies }\end{array}$ & $\begin{array}{l}\text { Different measures provide } \\
\text { different types of } \\
\text { information }\end{array}$ & $\begin{array}{l}\text { 1) Model accuracy better } \\
\text { tracked by the RSA than unit } \\
\text { activation (cf. BOLD)2) A } \\
\text { conceptually similar RSA } \\
\text { pattern to data in ref. } 71\end{array}$ \\
\hline
\end{tabular}

Chronic Aphasia after Left but Not Right Hemisphere Stroke. We next investigated whether damage to the left hidden layer in the model would be more likely to result in permanently impaired language performance (chronic aphasia) compared to damage to the right. Gajardo-Vidal et al. (17) found that approximately half of patients, $49 \%$ (151/307), with left hemisphere stroke showed impaired repetition performance, whereas the incidence for patients with right hemisphere stroke was about $5 \%(5 / 93)$. In the preceding section, we demonstrated that the model with more computational capacity in the left pathway produced a bilateral, left-asymmetric activation pattern similar to fMRI brain activations observed in most healthy individuals during language production. Thus, we opted to use a model with an asymmetrical structure where the computational capacity in the left was twice as large as that in the right (60 vs. 30 units). The 30 units in a hidden layer also met the minimum number of units required for a unilateral model to support the production task (SI Appendix, Fig. S1).

Fig. $2 A$ shows both the developmental learning trajectory before lesioning (the intact model) and an example recovery profile of the model with a left or right moderate lesion. During development, the model learned HF words more accurately and quickly than LF words. Generalization to NW was very good, although lower than performance on words. Then, a moderate lesion was applied to the left hidden $(\mathrm{LH})$ or right hidden $(\mathrm{RH})$ layer 1 in the model. A moderate lesion, $50 \%(0.5)$, meant that $50 \%$ of the units were damaged, and noise with the variance of 0.5 was added to the links connecting to and from the left hidden layer 1. After damage, the model was reexposed to its learning environment for 100,000 word presentations to allow for a period of experience-dependent, plasticity-related recovery (based on a reoptimization of the remaining processing units) (47). To mimic the loss of function and missing activation in the damaged brain regions immediately after stroke (50), a period of initial inefficient learning for the surviving units was implemented (i.e., their learning abilities were initially limited and then gradually regained, whereas, for the units in the unaffected layers, learning efficiency was normal). Inefficient learning was implemented by varying unit gain from zero to one in steps of 0.1 over the early stage of retraining (the first 10,000 word presentations in the recovery phase). Note that the model behaved similarly without the implementation of a period of inefficient learning (SI $\mathrm{Ap}$ pendix, Supplementary Simulations). Immediately after left damage, the performance of the model was at floor. Then, the model started to reoptimize the weight connections and relearned the task. In the later stage of recovery, performance gradually increased up to an asymptote (i.e., partial function recovery as found in chronic aphasia). In contrast, the right damage only caused minor disruptions to the performance, and it recovered rapidly (i.e., full function recovery akin to transient aphasia).

Obviously, patients have different lesion severities in the left or right hemisphere, leading to different recovery profiles. To capture this, different levels of damage were applied to the left or right hidden layer 1 . Specifically, 10 lesion levels were implemented by damaging hidden units from 10 to $100 \%$ with step increment of $10 \%$, plus adding Gaussian noise, with variance from 0.1 to 1 with step increment of 0.1 , to the links that were connected to and from the target hidden layer. All retraining procedures were the same as described above. Fig. $2 B$ shows the final recovered performance as a function of different levels of damage to the LH1 or RH1. For left lesions, the recovered performance varied with lesion levels. We divided the models into three lesion groups, $10 \%(0.1)$ to $30 \%$ (0.3) for the mild group, $40 \%(0.4)$ to $60 \%(0.6)$ for the moderate group, and $70 \%(0.7)$ to $100 \%$ (1) for 


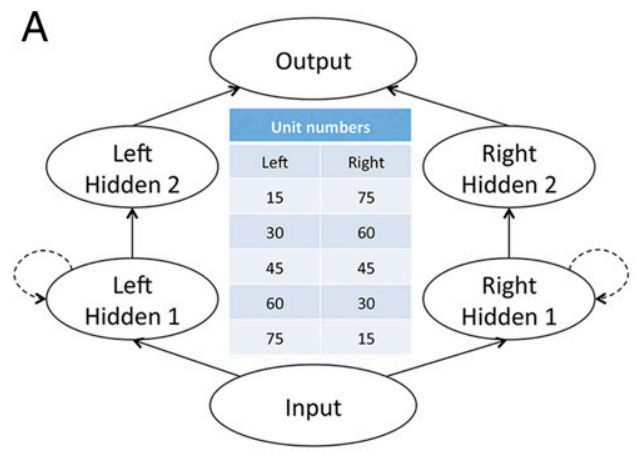

B

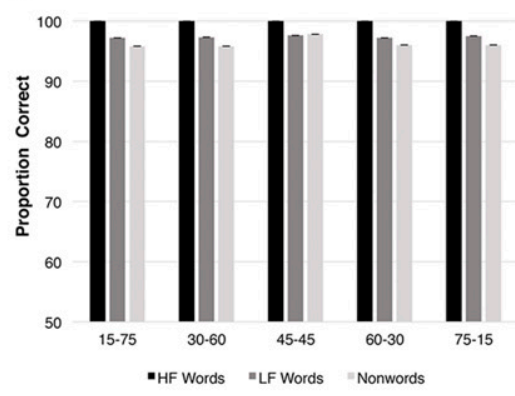

C

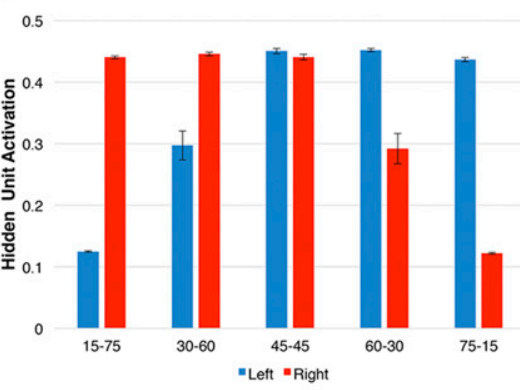

D

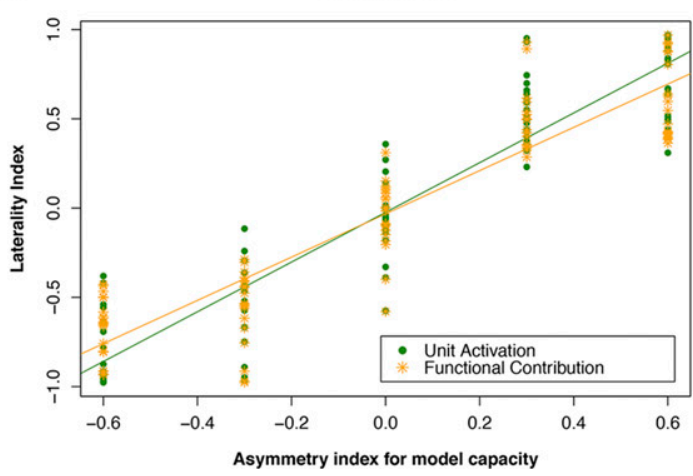

Behavioral Data (Reproduced from Vernooij et al. (2007))

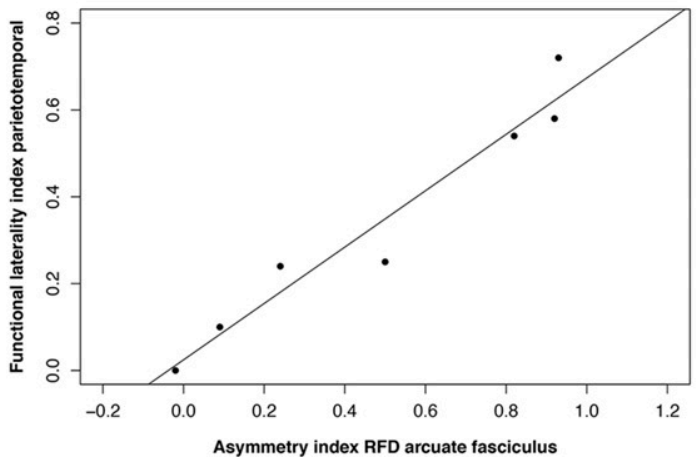

Fig. 1. The model architecture, repetition performance, average hidden unit activation, and lateralization patterns produced by the bilateral model with differential capacity in the left and right pathways. (A) The model with five different numbers of hidden units in the left and right pathways including 15 and 75, 30 and 60, 45 and 45, 60 and 30, and 75 and 15. The number of units in hidden 1 layer and hidden 2 layer was the same. The dashed lines indicate Elman connections (see Methods). (B) The repetition performance of the model on high-frequency words, low-frequency words, and nonwords. (C) Hidden unit activation produced by the model across the hidden layers along the left and right pathways. $(D)$ The lateralization patterns based on functional contribution and output unit activation produced by the model and the behavioral data reproduced from Vernooij et al. (42). HF, high frequency; LF, low frequency; RFD, relative fiber density. Error bar represents $\pm 1 \mathrm{SE}$.

the severe group. The mild group showed the best recovered performance, while the severe group was the worst, with the moderate group in the middle. For the right lesions, the model generally recovered very well regardless of lesion levels. These results demonstrated that, following damage and recovery, performance of the left-lesioned model was much more impaired than the right-lesioned model. The simulation data were generally consistent with the patients' studies reported in Gajardo-Vidal et al. (17), showing a stroke in the left hemisphere is more likely to lead to profound, chronic language impairment (Fig. 2B), albeit the right lesion model may have underestimated the mild level of aphasia that is sometimes observed (see Discussion).

Dynamic Activation Shifts in Poststroke Aphasia and Recovery. An important aspect of this study was to investigate the relationship between simulated behavioral performance and underlying metrics of unit function (to mimic functional neuroimaging data). Three levels of left lesions (20\% [0.2], 50\% [0.5], and 80\% [0.8]) were selected to simulate mild, moderate, and severe aphasia. Additionally, the severe right lesion (80\% [0.8]) was included to understand what compensated the effects of right damage. Four measures were used to reveal the mechanisms underlying recovery in the damaged model. First, as before, the damaged model's accuracy on word and nonword repetition was used to simulate poststroke aphasic patients' behavioral performance. Second, we used output unit activation in the left and right pathways as a proxy of fMRI activation (75). Additionally, we investigated whether the model could produce similar activation patterns to that observed by Saur et al. (50) during three phases of language recovery. Specifically, we investigated whether a mildly lesioned model could produce 1) from the acute phase to the subacute phase, an increase of the output unit activation in the undamaged left pathway and the right pathway with the highest increase in the right; and, 2) from the subacute phase to the chronic phase, a decrease of the output unit activation in the right pathway but with the output unit activation in the left remaining stable. Accordingly, the relearning time in the model was divided into three recovery periods (acute, subacute, and chronic) approximating different stages of patient recovery, and the average output unit activations were computed. Third, we measured the perilesional and contralateral hidden unit activations to examine which undamaged units in the model were reformulated to support during recovery. Lastly, we conducted representation similarity analysis (RSA) comparing the activation similarity patterns in the hidden layers to the output similarity for the words. To our knowledge, there is only one stroke patient study that has utilized RSA (71). This study found that, when reading, a patient with a severe lesion to the left visual word form area (VWFA) relied more on the right VWFA for orthographic processing, indexed by the RSA similarity scores, while the healthy participants generally relied more on the left VWFA than the right. As this investigation was a single case study of reading (not repetition), we report analogous data based on the closet settings to Fischer-Baum et al. (71), and investigated whether the reliance of the processing shifted from the left to right after severe damage to the left in the model.

In addition to these four measures, two additional measures were related to the model's relearning: average weight strength and weight change. Both measures were helpful for understanding 

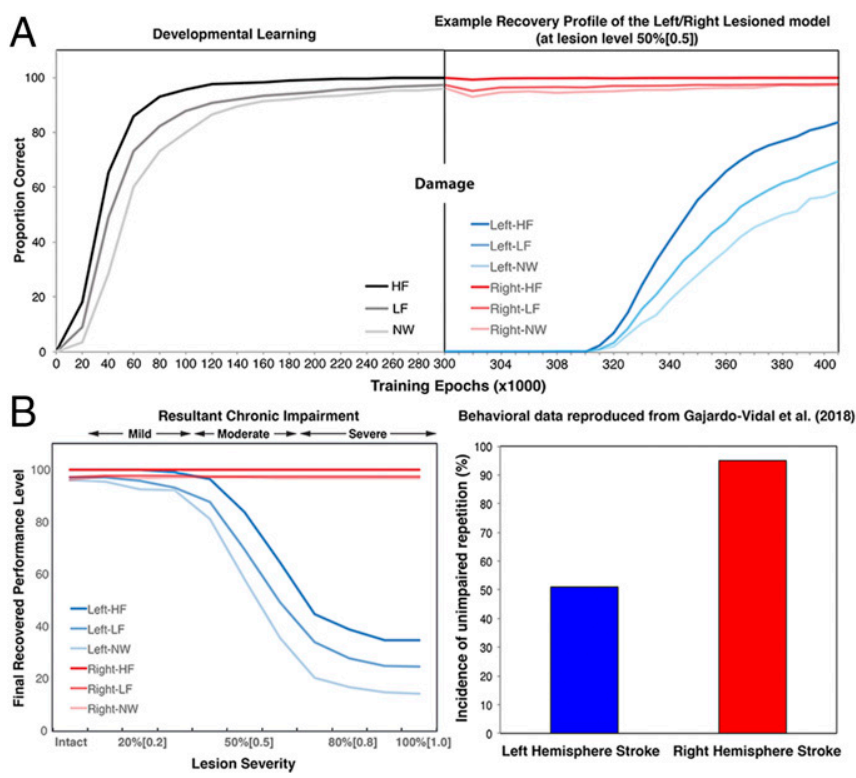

Fig. 2. (A) The developmental learning trajectory of the model before damage, and the recovery profile after damage (moderate lesion 50\% [0.5]) to the left or right hidden layer 1 , simulating a left or right hemisphere stroke and recovery. Note that the unequally spaced time scales for the relearning period were made to clearly demonstrate the model's relearning in different periods. $(B)$ The recovered performance of the left-lesioned model and the rightlesioned model as a function of lesion levels (a combination of unit damage and noise; see the Chronic Aphasia after Left but Not Right Hemisphere Stroke section for details). "Intact" means the model without lesion. The behavioral data of unimpaired repetition performance were reproduced from Gajardo-Vidal et al. (17). HF, high-frequency words; LF, low-frequency words; NW, nonwords; $\mathrm{LH}$, left hemisphere; $\mathrm{RH}$, right hemisphere.

how the model relearned the task during recovery and what the links were between recovery performance and relearning processes (see SI Appendix, Supplementary Simulations for details).

Fig. 3 summarizes several key phenomena. We can first look at performance accuracy and output unit activation. For the left lesion, the recovered performance of the model aligned with lesion severity, with the mild lesion model showing the best performance. Importantly, for the mildest lesion, there was a transient pattern of output unit activation shifting from left to right at the early stage of recovery and then back to left at the later stage of recovery, similar to the finding observed in the mild aphasic patients (50). To test these observations, we compared the simulation data against Saur et al. (50) by computing the differential output unit activations between the acute and the subacute phases, and between the subacute and the chronic phases. As can be seen in Fig. $4 A$, the output unit activations significantly increased for both the left and right pathways, $t=8.11, P<0.001$, and $t=8.56$, $P<0.001$, respectively, from the acute to the subacute phase. The increase of activation was numerically higher for the right than for the left pathway. The comparison of the subacute and chronic phases showed a significant decrease of activation in the right pathway, $t=-3.55, P=0.002$, but not in the left pathway $(P=$ $0.14)$. All of the statistics were corrected for multiple comparisons.

As for the moderate and severe lesion, the models in Fig. 3 showed right lateralized activation patterns, and the recovered performance was worse than that in the mild lesion. In contrast, even after a severe right lesion, accuracy was only slightly disrupted but quickly recovered, and the output activation pattern during recovery largely remained unchanged, with a small rise in right output unit activation.

We investigated how undamaged perilesional and contralesional units supported recovery. The results showed that, for both mild and moderate lesions, the LH1 perilesional activation initially decreased following damage but then gradually increased during relearning, reflecting a reoptimization process. A similar but larger initial decrement followed by a slower increment pattern was observed for LH2 hidden unit activation. For a severe lesion, both the LH1 and LH2 hidden unit activation decreased following damage but did not rise again, presumably because there were insufficient processing units available in the LH1 layer for the model to reoptimize. This pattern was also observed for the right severe lesion comparison, where both the RH1 perilesional activation and $\mathrm{RH} 2$ hidden unit activation gradually decreased and remained in a low activity level. Turning to contralateral activation, for all severities of the left lesion, the contralateral hidden unit activations at RH1 and RH2 increased very quickly following damage. The degree of increment was varied and depended on lesion severity, with the largest increment for the severe condition. By contrast, for the right severe condition, there was no clear increment of the contralateral hidden unit activations at LH1 and LH2.

For the correct interpretation of the relationship between patient behavioral performance and underlying activation, it may be important to note that there were differential associations between model accuracy and the various unit metrics. Fig. 3 shows that the RSA measure closely shadowed the changing model accuracy, quite unlike simple unit activation (a proxy to BOLD levels) which show a complex nonlinear relationship. Taking the left moderate lesion as an example, even when the right output unit activation was building up quickly during the initial recovery period, change in model performance was minimal. Subsequently, long after the point when the right output unit activation reached a relatively stable level, there was a much larger and gradual increase in model accuracy. By contrast, the change in the RSA pattern was closely aligned with model performance. Interestingly, although the right output unit activation was higher than the left output unit activation throughout recovery, the RSA results showed the left unit correlation was initially lower than the right unit correlation but returned to a higher level later in recovery.

To examine, formally, the relationships between model performance with output unit activation and the RSA measure, we conducted correlation analyses. Model performance was correlated with output unit activation and the RSA scores at LH1, $\mathrm{RH} 1, \mathrm{LH} 2$, and RH2 separately. Correlation analyses were conducted across the developmental learning period in the intact model and the relearning period in the lesioned model. Results are summarized in Table 2 . The correlations between output unit activation and model performance were mostly negative, in particular, for the lesioned conditions, except for the positive correlations for the left output unit activation in the intact condition and for the right output unit activation in the left severe lesion condition. When considering all intact and lesion conditions, the pattern of change in correlation for output unit activation was difficult to interpret. By contrast, the correlation with the RSA scores was more interpretable. The pattern of correlation change was moderated by lesion severity, revealing the sources of contribution to model performance. For the left lesions, left RSA unit correlations were much higher than the right RSA unit correlations in the milder lesion conditions. With increasingly severe lesions, the right RSA unit correlations increased with the decrease in the left RSA unit correlations. For the right severe condition, the left RSA unit correlations remained higher than the right RSA unit correlations. Fig. $4 B$ shows that the intact model produced a higher RSA unit correlation for the LH1 than the RH1, and the opposite pattern was found for the left severe model. These results were conceptually similar to the findings of Fischer-Baum et al. (71), in which the healthy controls relied more on the left VWFA for orthographic processing in contrast to the patient with severe damage to left VWFA relying more on the right VWFA. Obviously, 
Left
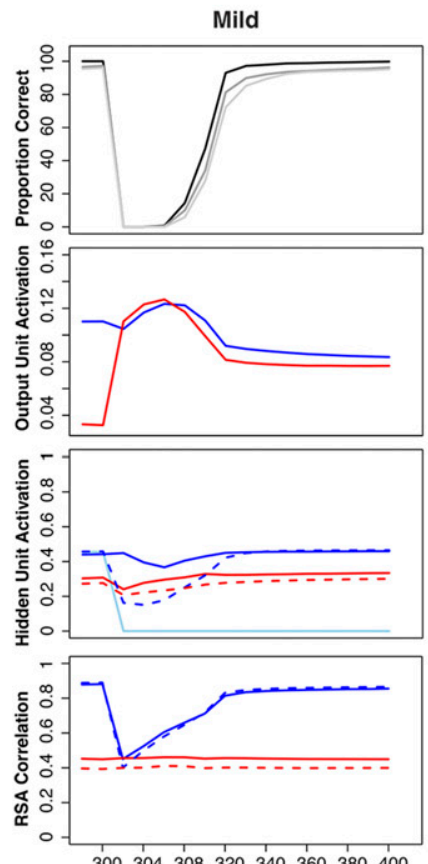

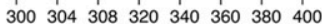

Moderate
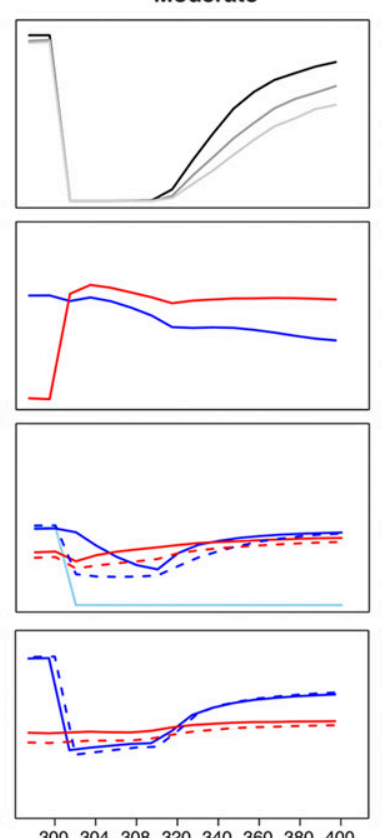

Training Epochs $(\mathbf{x} 1000)$

Severe
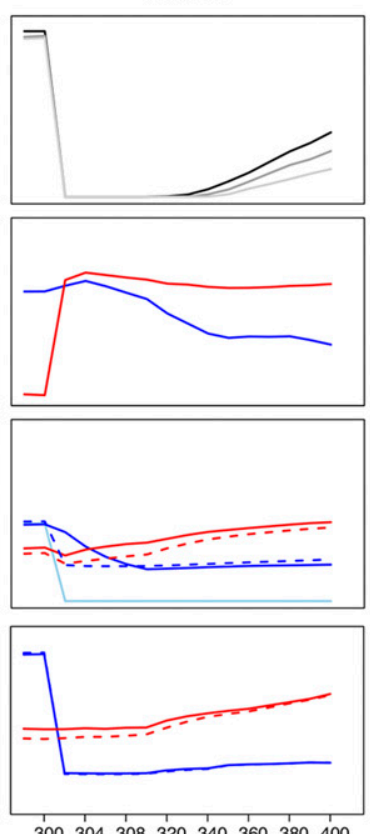

$\begin{array}{llllllll}300 & 304 & 308 & 320 & 340 & 360 & 380 & 400\end{array}$
Right
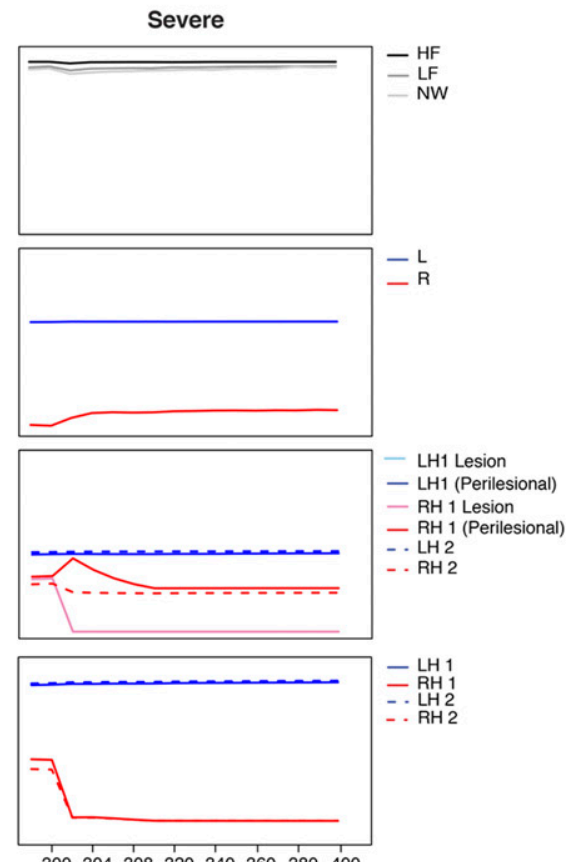

$\begin{array}{llllllll}300 & 304 & 308 & 320 & 340 & 360 & 380 & 400\end{array}$

Fig. 3. Simulation patterns of poststroke aphasia and recovery: left mild (20\% [0.2]), left moderate (50\% [0.5]), left severe (80\% [0.8]), and right severe (80\% [0.8]) conditions. The lesion level was a combination of the proportion (percent) of the units that was damaged and the range of noise (bracket) added to the connections to and from the hidden layer. For each lesion condition, the first panel shows model performance, the second panel shows output unit activation generated from the left and right pathway of the model separately, and the third panel shows hidden unit activation for the left and right hidden layers 1 and 2. The activations for lesioned and perilesional units are plotted separately; the fourth panel shows the RSA scores obtained in the left or right hidden layers 1 and 2 in the model. HF, high-frequency words; LF, low-frequency words; NW, nonwords; L, left; R, right; LH, left hidden layer; RH, right hidden layer.

the tasks in the present study and Fischer-Baum et al.'s study are different. Thus, the patterns produced by the model can be considered as predictions for future patient studies in spoken production. Collectively, these results demonstrated that the RSA could potentially provide a more direct measure to relate model performance to the underlying computations.

Interconnectivity Between the Left and Right Hemispheres. Thus far, the implemented model did not have interconnections between the left and right pathways. Cortical hemispheres, however, are connected by the corpus callosum as well as various subcortical routes (76). Given that the corpus callosum and interhemispheric connectivity are complex, a detailed neuroanatomically constrained simulation is beyond the scope of this study. However, we explored a simplified simulation by adding direct "homotopic" interconnections between the left and right pathways to investigate 1 ) whether this changed the patterns of simulated recovery reported above, and 2) whether the model would develop transcallosal inhibitory connectivity as proposed in various classical hypotheses $(6,7,9)$. Transcallosal connectivity in the model was implemented as sparse, bidirectional cross-connections between the left and right hemispheres without imposed positive or negative connections (all weight connections were allowed to develop freely). As there is no prior knowledge about interhemispheric connectivity density, we implemented two connectivity levels $(30 \%$ and $70 \%$ ). The training and testing procedures were exactly the same as previously described. We also ran additional comparison simulations in which the connections were constrained to be negative only (SI Appendix, Supplementary Simulations). This constrained model produced stronger left-lateralized patterns, and it was less resilient to damage. Analyses of the weight values demonstrated that the vast majority were close to zero; that is, the model effectively became one with independently functioning pathways. This was not true when the connection values were unconstrained.

Fig. 5 shows the resulting patterns produced by the left mild, left moderate, and left severe and right severe lesioned models with different levels of interconnection (unconstrained). For comparison, the pattern produced by the model without interconnections is included in Fig. 5. Overall, the patterns were similar. There was transient right unit activation for the left mild lesion condition but not for more-severe left lesion conditions. In addition, the model could recover to a similar accuracy level regardless of the levels of interconnection. But, when the model had more interconnections, it showed a more bilateral pattern following damage and recovery, increasingly behaving like a single functional pathway model. This observation was confirmed by the results from the right severe lesion condition, where the model with more interconnections exhibited a more pronounced impairment in the early recovery phase.

\section{Discussion}

Understanding the brain mechanisms underlying language processing is critical, both theoretically and clinically. To tackle various key issues that appear to be contradictory in healthy and impaired language processing (Table 1), we developed a single, unified neurocomputational model of spoken language production with bilateral pathways. The key features of this modeling work include the importance of considering healthy and impaired language within an intrinsically bilateral but asymmetric language network; to conceptualize recovery of function after damage as an experience-dependent plasticity-related learning process; and to provide a platform to simulate behavioral and neuroimaging data from different populations. A list of the key findings is provided in Table 1 , and each are discussed briefly below. 

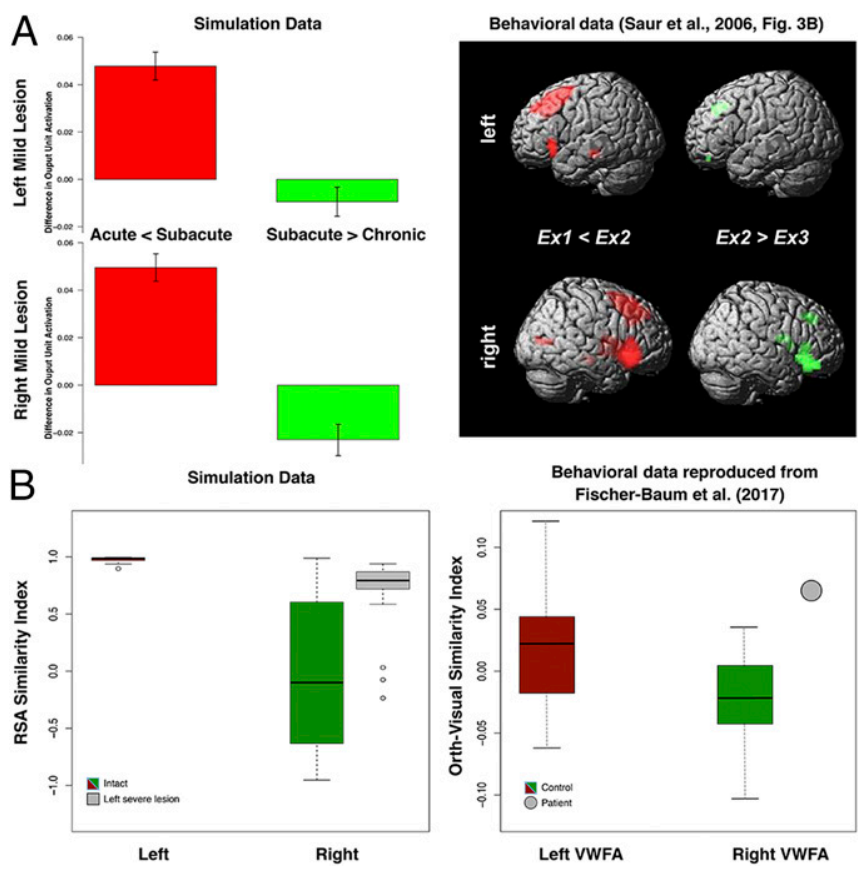

Fig. 4. (A) The increment and decrement of the activations in the mode with a left mild lesion. Direct comparisons of output unit activations between the acute $(300 \mathrm{~K}$ to $301 \mathrm{~K}$ ) and subacute $(301 \mathrm{~K}$ to $305 \mathrm{~K})$ phases and between the subacute and chronic ( $305 \mathrm{~K}$ to $340 \mathrm{~K}$ ) phases with the fMRI data of up-regulation and down-regulation in the language network reported by Saur et al. (50). Ex1, acute; Ex2, subacute; Ex3, chronic. (B) RSA similarity indices of the left hidden layer 1 for both the intact model and the model with a severe left lesion. The orth-visual similarity indices for both the controls and the patient in the left WWFA and the right WWFA are from Fischer-Baum et al. (71).

In an otherwise computationally homogenous model, an initial imbalance in the processing capacity (number of hidden units) in the left and right hemisphere pathways was sufficient to explain the pattern of data observed in healthy participants and patients with chronic aphasia. Specifically, the capacity imbalance drives an emergent division of labor across the pathways such that the left hemisphere pathway picks up more of the computational work (i.e., each unit, on average, is more highly activated and contributes more to the final spoken output response than each corresponding right hemisphere unit). As a result, the undamaged model shows bilateral but asymmetric "activation" as observed in healthy participants. When this capacity imbalance is combined with plasticity-related recovery, the model provides an explanation for why left hemisphere stroke is more likely to result in chronic aphasia than right hemisphere stroke. Plasticity-related recovery reflects a reoptimization of the remaining connection weights to maximize behavioral performance. This occurs in both "perilesional" units and the contralateral pathway. The greater computational capacity in the left hemisphere means that, when the right hemisphere is damaged, there is greater capacity for the left hemisphere pathway to pick up the extra representational work previously undertaken by the (damaged) right hemisphere pathway (meaning that there is only transient aphasia). The same recovery process occurs following left hemisphere damage, except that 1) the greater left hemisphere capacity means that, at least for mild levels of damage, there is still enough spare capacity in the remaining ipsilateral units to pick up the additional computational work (i.e., there is good or recovered function, and left hemisphere activation still dominates, even after mild levels of left hemisphere damage) and 2) there is insufficient capacity in the right hemisphere to compensate completely if the left hemisphere damage is too severe. In such circumstances, the model mimics chronic aphasia. In all cases, plasticity-related recovery means that there is a dynamic shift in the division of labor to ipsilateral "perilesional" and contralateral areas, as is observed in fMRI studies of recovered patients. The model also demonstrates that there can be complex, nonlinear relationships between behavioral performance and levels of unit activation (a proxy for BOLD), whereas the relationship is much more direct when comparing performance to the accuracy of the representations coded in the pathway (implying that MVPA-type neuroimaging analyses may be a better way to assess and track the neural bases of recovery in aphasic patients).

Leftward hemispheric asymmetry has been shown in several brain regions and white matter tracts (36-40). However, there remains some controversy regarding a positive correlation between structural asymmetry and functional lateralization $(25,41$, $42,77)$. The discrepancy could be related to individual differences among participants (e.g., age, education, handedness, and gender), or it could be because most studies have relatively small sample sizes (78). In a more controlled computational environment, our bilateral model with differential pathway revealed the impact of model capacity on the functional division of labor underlying performance and demonstrated a link between hemispheric asymmetry and language lateralization. The simulation results are consistent with the positive correlation patterns reported in most right-handed healthy participants $(41,42)$. The model also shows that this structural difference could be fundamentally important for explaining patient data. By explicitly incorporating a leftward asymmetric but bilateral structure in the model, the model synthesizes the seemingly contradictory patterns observed in both healthy participants and aphasic patients (Fig. 2): specifically, a leftward asymmetric but bilateral pattern in the intact model, and the much stronger lateralization picture that is observed in chronic patients after left (aphasic) vs. right (recovered) lesions. Additionally, the relationship between the severity of the left lesion and recovered performance is nonlinear (Fig. $2 B$ ), suggesting that the model had developed some resilience to mild damage (up to $235 \%$ ) but, beyond a "tipping point," the effects of damage cannot be overcome through plasticity-related relearning, leading to more permanent language impairment as observed in chronic aphasia. It is important to note that there was a small divergence between the simulation results and GajardoVidal et al.'s (17) patient data; that is, the model is more robust to right hemisphere damage (Fig. $2 B$ ). It is possible that this highlighted version of the model might possess a division of labor too biased to the left hemisphere pathway, making the contribution of the right hemisphere a little too weak. From our explorations of the key computational parameters, we know that the division of labor is governed in part by 1) the asymmetry in the balance of computational resources (Fig. 1) and 2) the degree of interconnectivity between the left and right pathways (Fig. 5).

Taking a step back, this explanatory framework for aphasia and recovery raises some fascinating fundamental questions about higher cognition more generally: 1) Why is it good for cognitive functions to be supported bilaterally, and, 2) if so, why is it beneficial for some functions to remain at least partially dominated by one hemisphere (compare to the asymmetric yet bilateral architecture of the language production system)? Complete answers to these questions will have to wait for future research, but there are some initial ideas in the literature. With regards to the benefits of bilateral implementation, a recent computational model and formal mathematical analysis demonstrated that bilateral systems are much more robust to the effects of damage than is a singular system with the same resources (79). The second question is more difficult to answer. One possibility is that a bilateral system's resources might be pulled asymmetrically if that cognitive function has to interact with other computations/ representations that, themselves, are unilaterally expressed (although, of course, this begs the same question of why these are 
Table 2. The correlations between model performance and output unit activations and RSA scores across the developmental learning period in the intact model and the relearning period in the lesioned models

Intact $\mathrm{L}$ mild $\mathrm{L}$ moderate $\mathrm{L}$ severe $\mathrm{R}$ severe

L output unit act $0.23 * * *-0.29 * * * \quad-0.20 * * * \quad-0.1 * \quad-0.23 * * *$

R output unit act $-0.48^{* * *}-0.37 * * * \quad-0.04 \quad 0.09 *-0.06$

$\begin{array}{llllll}\text { L RSA H2 } & 0.84 * * * & 0.92 * * * & 0.82 * * * & 0.33 * * * & 0.42 * * *\end{array}$

$\begin{array}{llllll}\text { R RSA H2 } & -0.08 & -0.04 & 0.40 * * * & 0.69 * * * & -0.08\end{array}$

$\begin{array}{llllll}\text { L RSA H1 } & 0.82 * * * & 0.92 * * * & 0.81 * * * & 0.29 * * * & 0.44 * * *\end{array}$

\begin{tabular}{lllll} 
R RSA H1 & -0.04 & -0.05 & $0.28 * * *$ & $0.64 * * *$ \\
\hline
\end{tabular}

$P<0.05 ; * * * P<0.001 ; \mathrm{L}$, left; $\mathrm{R}$, right; act, activation.

asymmetrically supported). A second possibility comes from a potential downside of distributing the same function across multiple brain regions. While the distributed system may engender greater robustness to the effects of damage, it might induce a need for heightened synchronization. Previous proposals (80) noted that human connected speech is highly demanding in terms of the rapid, accurate motor executions required, as well as the fast conceptual-to-speech transformations (81). When output signals need to change rapidly and accurately, then, in the situation of bilateral systems, the need for synchronization also increases (80). In the limit, sufficient synchronization across distributed brain regions may be impossible to achieve, and thus the "compromise" is to let one side of the computation dominate; that is an asymmetric, bilateral system.

Two potential mechanistic frameworks have been proposed for language recovery: degeneracy and variable neurodisplacement (13). Both mechanisms allow the language system to be at least partially resilient to damage and for recovery of function. Recovery can be accomplished by a permanent reformulation of the remaining multiple codes (degeneracy) or up-regulation of systems (variable neurodisplacement), or both. The present neurocomputational model demonstrates that both mechanisms
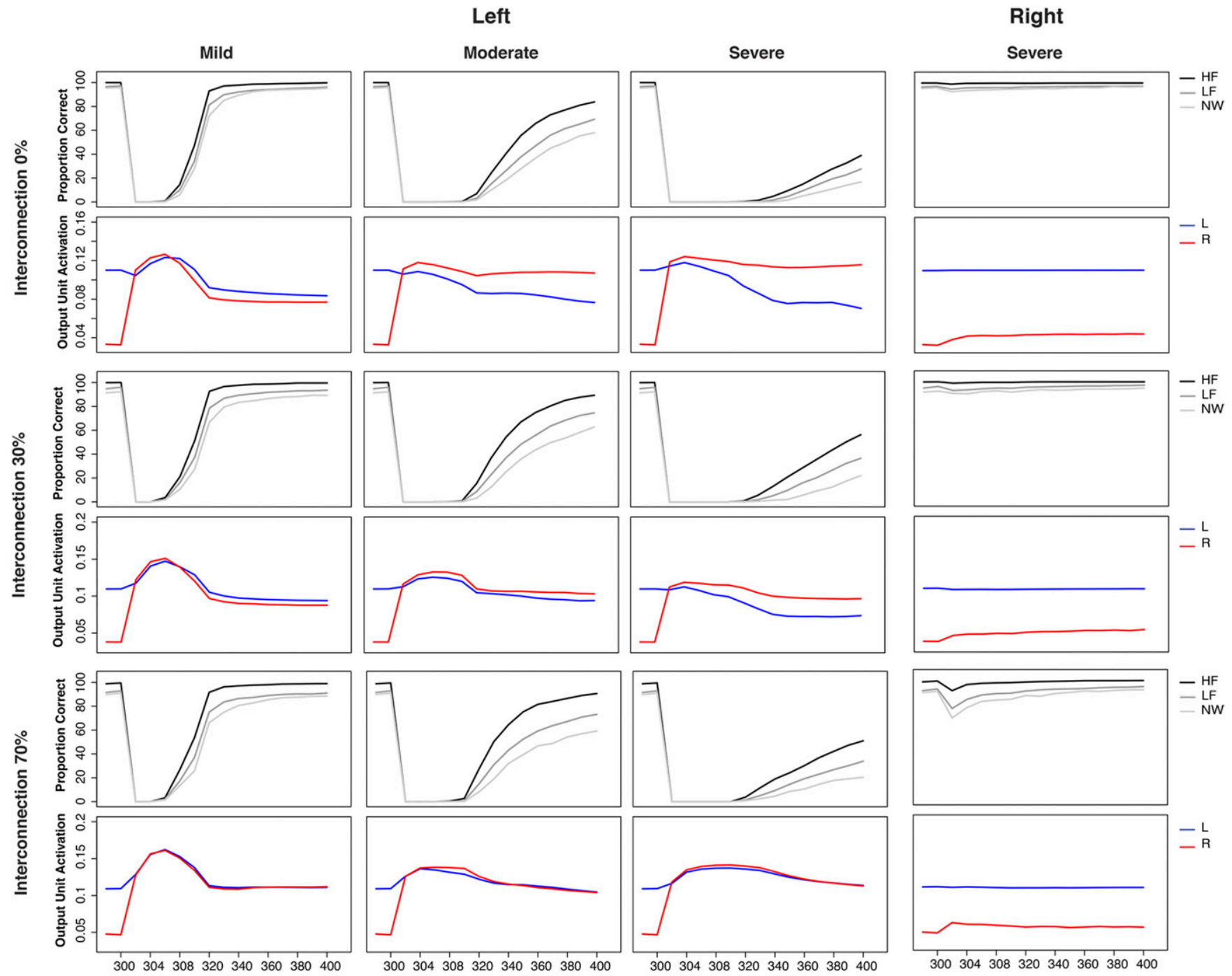

Training Epochs (x1000)

Fig. 5. Simulation patterns of poststroke aphasia and recovery produced by the model with three levels of interconnections ( $0 \%$, $30 \%$, and $70 \%)$ between left and right sides for the left mild (20\% [0.2]), left moderate (50\% [0.5]), left severe ( $80 \%$ [0.8]), and right severe (80\% [0.8]) lesion conditions. The lesion level was a combination of the proportion (percent) of the units that was damaged and the range of noise (bracket) added to the connections to and from the hidden layer. For each lesion and interconnection conditions, Top shows model performance and Bottom shows output unit activation generated from the left and right pathway of the model separately. HF, high-frequency words; LF, low-frequency words; NW, nonwords; L, left; R: right. 
are not mutually exclusive, and they can be utilized as a part of the recovery process. Immediately after dominant pathway damage, the model rapidly activates contralesional activation and also starts to reformulate the perilesional unit contributions. If the perilesional units are capable of resupporting the function, then, later in recovery, both perilesional and contralateral contributions increase; otherwise, the perilesional contribution is decreased, and the enhanced contralateral contribution continues. As such, it would appear that the recovery process follows the two proposed principles, but the actual mechanisms involved depend on the level of task engagement by the units before damage and whether there is sufficient capacity in the remaining perilesional or contralateral areas to support recovery. As a result, there are differential output activation recovery profiles depending solely on lesion severity. With a mild left lesion, the perilesional units are largely persevered and can be reformulated for recovery, leading to good recovery and left-lateralized output activation patterns. With a more severe left lesion to the model, perilesional support is reduced, and partial recovery relies mainly on the contralateral units. Accordingly, there is a cooccurrence of slow and imperfect recovered performance with right-lateralized activation patterns. These simulations collectively mirror the patient results reported in the literature: Good performance is associated with leftlateralized activations, while worse performance is associated with more right-lateralized activations (5), and left-right-left changing brain activation patterns are observed in patients with mild brain lesions in the left hemisphere (50). This finding emphasizes the importance of considering lesion severity when interpreting associations between good recovery and left-lateralized brain activation patterns $(5,50)$ and the association between imperfect recovery and right-lateralized brain activation patterns (82).

The present bilateral model also provides a potential explanation for why the right hemisphere provides some but not perfect language support. The classical RHH proposes that the right hemisphere is normally suppressed, via transcallosal inhibition, by the dominant left hemisphere system, but it can be released to provide some function after significant left hemisphere damage (6, $7,9)$. As noted previously $(2,5,8,10-13,27,50,56)$, the RHH leaves many puzzling questions open, including how the right hemisphere can develop language representations under lifelong suppression, how left and right language systems might contribute to normal function, what bilateral yet asymmetric BOLD activation in healthy participants represents, and why this biologically expensive organization for all people is an optimal solution for the minority of people who happen to suffer from the right kind of brain damage to induce aphasia. The current simulations provide a much more straightforward proposal for the data. The premorbidly bilateral albeit asymmetric system supports healthy function but can partially reoptimize following damage. This can all be achieved without any recourse to notions of juvenile right hemisphere language systems and interhemispheric inhibition. Instead, the right hemisphere subsystem is less efficient because it has less computational capacity, and, in turn, learning in the left hemisphere overshadows that in the right, resulting in the left hemisphere units taking up more of the representational work (Fig. 1C). These results follow even without interhemispheric connection. Even if included (Fig. 5), then 1) they do not all become inhibitory and, 2) with increasing connectivity, the model evolves into a single functional system. Of course, it should be acknowledged that the connections within corpus callosum are much more complex than the simple parallel connections implemented in the present model. While interhemispheric connectivity has been shown to be inhibitory within the motor network (62-64), to our knowledge, there is currently no evidence of transcallosal inhibitory in language or other higher cognitive networks; in contrast, a few studies have demonstrated interhemispheric excitatory connectivity $(65,66)$. Finally, in a third variant in which inhibitory-only interhemispheric connections were enforced, the model set their value close to zero.

We should note that one previous study (9) applied TMS to left IFG in healthy participants during a verbal fluency task, and showed decreased brain activity in the left but increased activity in the right homolog. These findings were interpreted as supportive evidence for transcallosal inhibition from the left to right hemispheres; however, the changes in the effective connectivity between the left and right inferior frontal gyri after TMS were not examined. Alternatively, the up-regulation of homolog language areas after brain stimulation could be considered as a form of adaptive plasticity based on an interhemispheric compensatory mechanism $(31,32,35,83)$. For example, a recent study of semantic processing, combining theta-burst stimulation (cTBS) and dynamic causal modeling (DCM) (32) found increased right ventral anterior temporal lobe (vATL) in response to cTBS to the left vATL. The DCM results revealed an increase in the facilitatory drive from the right to the left vATL. There was no evidence of negative inter-ATL connectivity with or without stimulation. Similar results have been reported in another brain stimulation study targeting Broca's area during speech processing (35).

Lastly, the model investigated multiple measures within a single framework and their sometimes complex relationships. The simulation results suggest that, in task-based fMRI studies, BOLD signals and RSA measures may provide different information: Although increase unit activations (compare to BOLD increases) are a necessary precursor to behavioral recovery, higher unit activations do not necessarily imply that the units are contributing to improved performance. In the model, the performance improvement required both increasing unit activation and tuning weight connections. Immediately after damage, the activation level of the units in the model was generally low. Thus, the first step toward relearning was to increase the activation level via a generalized weight connection increase. This was followed by retuning weight connections in order to minimize the errors between the target and actual patterns at the output layer. The implication is that fMRI BOLD signals in patients during recovery have an ambiguous interpretation; they could reflect the neural basis for recovered performance (as occurs, for example, in the right hemisphere pathway after severe left hemisphere lesions: BOLDtype and then RSA-type measures increase; Fig. 3) or, alternatively, generalized but untuned activation (e.g., after a moderate lesion: Right hemisphere activation increases but performance recovers only after the left hemisphere RSA has bounced back; Fig. 3). In contrast, RSA might provide a more direct measure to link recovered performance with neuronal pattern information in different phases of aphasia recovery. This result is consistent with a growing interest in using different types of neuroimaging analyses to investigate the right hemisphere activation patterns in poststroke aphasia and how it is related to recovered performance $(70,71)$. Given that, to date, there have not been any RSA-based studies of spoken language production in aphasic patients, the current simulations serve as model predictions for future neuroimaging studies. By extension, the same techniques might also be helpful in clarifying the (dis) advantages of using brain stimulation techniques (TMS or transcranial direct current stimulation [tDCS]) to alter brain activation for effective treatments.

To finish, we note, explicitly, that there were at least three deliberate simplifications adopted in the model which can be addressed in future work. First, the model focused on speech production along the dorsal pathway. Obviously, there are multiple pathways in the language network $(16,40,84-87)$. For example, we have not considered the ventral pathway that includes a semantic system for comprehension. A previous neurocomputational model (46) demonstrated that a dual-pathway neural network model could simulate different types of aphasia (including receptive 
and expressive language) based on damage to a corresponding lesion site. Secondly, the psycholinguistic and cognitive science literatures contain many sophisticated models of individual language tasks (typically in relation to healthy performance) that embrace large word corpora and linguistic detail. We deliberately adopted a much more psycholinguistically simple model so that we could more easily explore the potential relationship between behavioral performance and brain structures/pathways, and thus allow us to distil some principal mechanisms relating to the emergent division of labor between the two hemispheres and how this can change in recovery. Third, we acknowledge that the backpropagation algorithm has commonly been viewed as biologically problematic even though it is the most efficient and effective for deep neural networks (88). However, this view may be changing. A very recent review (89) proposed that there could be a more direct neural analog of backpropagation: In simple terms, top-down feedback/prediction could be compared at the local level against the bottom-up sensory input. Clearly, however, more empirical evidence connecting the backpropagation algorithm and learning in the brain is needed. Future models can merge and elaborate these approaches to provide further systematic investigations, thereby elucidating the neural bases of healthy language and partial recovery in poststroke aphasia.

\section{Methods}

Model Architecture. The bilateral model was implemented as a simple recurrent network. The dual-pathway architecture of the model is shown in Fig. $1 A$. Each processing pathway consisted of two hidden layers. For the first hidden layer, there was a copy of the hidden layer, shown as dashed lines in Fig. 1A, known as an Elman layer (90); this allows a copy of the hidden layer from the previous time tick to influence the current unit activations, functioning as a memory buffer in the model.

The input phonological layer was connected to the first left and right hidden layers with Elman connections and then to the second left and right hidden layers and then to the single, final output layer. All layers were fully connected, and the connections were unidirectional unless stated otherwise.

Representation. Both 100 three-phoneme high-frequency and 100 threephoneme low-frequency monosyllabic words with consonant-vowel-consonant structures were included in the training set. Each word was represented by three phoneme slots, with each slot consisting of 25 phonetic features $(91,92)$ (for details, see SI Appendix, Supplementary Methods). The nonword list comprised 25 items created by changing the first consonant, the vowel, or the final consonant in a word.

Training and Testing. The model was trained on word repetition. For each word, the model was run for six time ticks. In the first three time ticks, each phoneme was presented in the input layer sequentially. There was no output target until all of the phonemes were presented. From the fourth time tick to the sixth time tick, the model was required to produce the target phonemes sequentially. Which word was presented to the model was determined by its logarithmic frequency (93). The model was trained using a standard

1. S. T. Engelter et al., Epidemiology of aphasia attributable to first ischemic stroke: Incidence, severity, fluency, etiology, and thrombolysis. Stroke 37, 1379-1384 (2006)

2. T. Barlow, On a case of double hemiplegia, with cerebral symmetrical lesions. BMJ 2 103-104 (1877).

3. P. Broca, Sur le siège de la facultlé du language articulé. Bull. Soc. Anthropol. 6, 337-393 (1865)

4. C. Wernicke, Der Aphasische Symptomencomplex, Eine Psychologische Studie auf Anatomischer Basis (Cohn and Weigert, Breslau, Germany, 1874).

5. W. D. Heiss, J. Kessler, A. Thiel, M. Ghaemi, H. Karbe, Differential capacity of left and right hemispheric areas for compensation of poststroke aphasia. Ann. Neurol. 45, 430-438 (1999).

6. W. D. Heiss, A. Thiel, A proposed regional hierarchy in recovery of post-stroke aphasia. Brain Lang. 98, 118-123 (2006).

7. H. Karbe, K. Herholz, M. Halber, W. D. Heiss, Collateral inhibition of transcallosal activity facilitates functional brain asymmetry. J. Cereb. Blood Flow Metab. 18, 1157-1161 (1998)

8. H. J. Rosen et al., Neural correlates of recovery from aphasia after damage to left inferior frontal cortex. Neurology 55, 1883-1894 (2000)

9. A. Thiel et al., Direct demonstration of transcallosal disinhibition in language networks. J. Cereb. Blood Flow Metab. 26, 1122-1127 (2006).

10. D. Cardebat et al.; PET Language Activation Study, Behavioral and neurofunctiona changes over time in healthy and aphasic subjects: A PET language activation study. Stroke 34, 2900-2906 (2003). backpropagation algorithm. Weight connections were updated after each word presentation on the basis of cross-entropy error

All words were used for training, and the nonword list was used for testing generalization. The model was trained for 300,000 word presentations, at which point the model repeated words accurately $(>98 \%)$ and generalized to nonwords ( $>96 \%$ ) (Fig. 2A). The model's performance was assessed by comparing the output phonological pattern with the sequential phoneme target. The model was judged to be correct only when all phonemes were correct. Twenty versions of the model with different random initial weights were trained to simulate different participants and to prevent the results emerging from idiosyncratic random initial weights. More detailed training environment and testing procedures are reported in SI Appendix, Supplementary Methods.

Neuroimaging Correlates. In neuroimaging studies, a laterality index was computed by subtracting the signal obtained in the right language areas from the corresponding left language areas and then dividing the score by the sum of the signals $(42,73)$. For the simulation, two measures were used: One was functional correlation $(47,74)$ as a proxy of effective connectivity signals, and the other was output unit activation (75), as a proxy of fMRI activation. For functional correlation, we recorded the unique contribution from the left pathway to the output phonological layer for all words (from the fourth to the sixth time ticks). This was achieved by lesioning the links between input and the $\mathrm{RH} 1$ so that there was no input signal from the right pathway. Similarly, the right pathway contribution was obtained by lesioning the links between input and the LH1. We then correlated the activation patterns from each pathway with the patterns when both pathways were utilized. For output unit activation, the same lesioning technique was used to isolate the unique contributions from the left or right pathway to the output layer activations. Both measures were used to compute the lateralization index (a positive score indicates a left-lateralized pattern).

RSA. For each word, the model produced three phonemes sequentially. To conduct RSA (95), we concatenated the three output activations into one output pattern for each word. We then computed a target representational dissimilarity matrix (RDM) based on the correlation distance of all word pairs. Similarly, for model RDMs, we computed the matrices based on the correlation distance of hidden unit activation patterns at LH1, RH1, LH2, and LH2 in the model independently. The hidden unit activation pattern for each word consisted of hidden unit activations of the constituent phonemes. The RSA correlation scores between the target RDM and the model RDMs of hidden unit activations were reported.

Data Availability. The datasets generated and analyzed in this study and the computer scripts used to run the simulations and to analyze the results are available on the MRC Cognition and Brain Sciences Unit Data Repository (https://www.mrc-cbu.cam.ac.uk/bibliography/opendata/).

ACKNOWLEDGMENTS. This research was supported by an European Research Council Advanced Grant (GAP 670428 - BRAIN2MIND_NEUROCOMP) to M.A.L.R. and Medical Research Council intramural funding program (MC_UU_00005/18).

11. J. Crinion, C. J. Price, Right anterior superior temporal activation predicts auditory sentence comprehension following aphasic stroke. Brain 128, 2858-2871 (2005).

12. L. M. Skipper-Kallal, E. H. Lacey, S. Xing, P. E. Turkeltaub, Functional activation independently contributes to naming ability and relates to lesion site in post-stroke aphasia. Hum. Brain Mapp. 38, 2051-2066 (2017).

13. J. D. Stefaniak, A. D. Halai, M. A. Lambon Ralph, The neural and neurocomputational bases of recovery from post-stroke aphasia. Nat. Rev. Neurol. 16, 43-55 (2020).

14. G. H. Eggert, Wernicke's Works on Aphasia. A Sourcebook and Review (Mouton, The Hague, The Netherlands, 1977).

15. N. Geschwind, Current concepts: Aphasia. N. Engl. J. Med. 284, 654-656 (1971)

16. L. Lichtheim, On aphasia. Brain 7, 433-484 (1885).

17. A. Gajardo-Vidal et al., How right hemisphere damage after stroke can impair speech comprehension. Brain 141, 3389-3404 (2018).

18. M. A. Lambon Ralph, L. Cipolotti, F. Manes, K. Patterson, Taking both sides: Do unilateral anterior temporal lobe lesions disrupt semantic memory? Brain 133, 3243-3255 (2010).

19. M. A. Lambon Ralph, S. Ehsan, G. A. Baker, T. T. Rogers, Semantic memory is impaired in patients with unilateral anterior temporal lobe resection for temporal lobe epilepsy. Brain 135, 242-258 (2012)

20. M. A. Lambon Ralph, J. L. McClelland, K. Patterson, C. J. Galton, J. R. Hodges, No right to speak? The relationship between object naming and semantic impairment: Neuropsychological evidence and a computational model. J. Cogn. Neurosci. 13, 341-356 (2001). 
21. J. R. Binder et al., Human temporal lobe activation by speech and nonspeech sounds. Cerebral Cortex 10, 512-528 (2000).

22. M. Bozic, L. K. Tyler, D. T. Ives, B. Randall, W. D. Marslen-Wilson, Bihemispheric foundations for human speech comprehension. Proc. Natl. Acad. Sci. U.S.A. 107, 17439-17444 (2010).

23. G. B. Cogan et al., Sensory-motor transformations for speech occur bilaterally. Nature 507, 94-98 (2014)

24. M. Mion et al., What the left and right anterior fusiform gyri tell us about semantic memory. Brain 133, 3256-3268 (2010).

25. D. Poeppel, The neuroanatomic and neurophysiological infrastructure for speech and language. Curr. Opin. Neurobiol. 28, 142-149 (2014).

26. P. E. Turkeltaub, H. B. Coslett, Localization of sublexical speech perception components. Brain Lang. 114, 1-15 (2010).

27. S. C. Blank, S. K. Scott, K. Murphy, E. Warburton, R. J. S. Wise, Speech production Wernicke, Broca and beyond. Brain 125, 1829-1838 (2002).

28. B. Mazoyer et al., Gaussian mixture modeling of hemispheric lateralization for language in a large sample of healthy individuals balanced for handedness. PLoS One $\mathbf{9}$, e101165 (2014)

29. D. Zacà et al., Whole-brain network connectivity underlying the human speech articulation as emerged integrating direct electric stimulation, resting state $\mathrm{fMRI}$ and tractography. Front. Hum. Neurosci. 12, 405 (2018).

30. C. J. Price, K. J. Friston, Degeneracy and cognitive anatomy. Trends Cogn. Sci. 6 416-421 (2002)

31. R. J. Binney, M. A. Ralph, Using a combination of $f M R I$ and anterior temporal lobe rTMS to measure intrinsic and induced activation changes across the semantic cognition network. Neuropsychologia 76, 170-181 (2015).

32. J. Jung, M. A. Lambon Ralph, Mapping the dynamic network interactions underpin ning cognition: A cTBS-fMRI study of the flexible adaptive neural system for semantics. Cereb. Cortex 26, 3580-3590 (2016).

33. M. A. Lambon Ralph, G. Pobric, E. Jefferies, Conceptual knowledge is underpinned by the temporal pole bilaterally: Convergent evidence from rTMS. Cereb. Cortex 19, 832-838 (2009).

34. G. Pobric, M. A. Lambon Ralph, E. Jefferies, The role of the anterior temporal lobes in the comprehension of concrete and abstract words: rTMS evidence. Cortex 45 1104-1110 (2009)

35. G. Hartwigsen et al., Perturbation of the left inferior frontal gyrus triggers adaptive plasticity in the right homologous area during speech production. Proc. Natl. Acad. Sci. U.S.A. 110, 16402-16407 (2013).

36. M. Catani et al., Symmetries in human brain language pathways correlate with verba recall. Proc. Natl. Acad. Sci. U.S.A. 104, 17163-17168 (2007).

37. M. Catani, M. Mesulam, The arcuate fasciculus and the disconnection theme in language and aphasia: History and current state. Cortex 44, 953-961 (2008)

38. R. Dorsaint-Pierre et al., Asymmetries of the planum temporale and Heschl's gyrus: Relationship to language lateralization. Brain 129, 1164-1176 (2006).

39. N. Geschwind, W. Levitsky, Human brain: Left-right asymmetries in temporal speech region. Science 161, 186-187 (1968).

40. G. J. Parker et al., Lateralization of ventral and dorsal auditory-language pathways in the human brain. Neuroimage 24, 656-666 (2005).

41. S. Ocklenburg, K. Hugdahl, R. Westerhausen, Structural white matter asymmetries in relation to functional asymmetries during speech perception and production. Neuroimage 83, 1088-1097 (2013)

42. M. W. Vernooij et al., Fiber density asymmetry of the arcuate fasciculus in relation to functional hemispheric language lateralization in both right- and left-handed healthy subjects: A combined fMRI and DTI study. Neuroimage 35, 1064-1076 (2007)

43. A. M. Findlay et al., Dynamics of hemispheric dominance for language assessed by magnetoencephalographic imaging. Ann. Neurol. 71, 668-686 (2012).

44. T. Traut et al., MEG imaging of recurrent gliomas reveals functional plasticity of hemispheric language specialization. Hum. Brain Mapp. 40, 1082-1092 (2019).

45. J. S. Bain, J. D. Yeatman, R. Schurr, A. Rokem, A. A. Mezer, Evaluating arcuate fasciculus laterality measurements across dataset and tractography pipelines. Hum. Brain Mapp. 40, 3695-3711 (2019)

46. T. Ueno, S. Saito, T. T. Rogers, M. A. Lambon Ralph, Lichtheim 2: Synthesizing aphasia and the neural basis of language in a neurocomputational model of the dual dorsalventral language pathways. Neuron 72, 385-396 (2011).

47. S. R. Welbourne, A. M. Woollams, J. Crisp, M. A. Ralph, The role of plasticity-related functional reorganization in the explanation of central dyslexias. Cogn. Neuropsychol. 28, 65-108 (2011)

48. D. Attwell, S. B. Laughlin, An energy budget for signaling in the grey matter of the brain. J. Cereb. Blood Flow Metab. 21, 1133-1145 (2001).

49. W. A. Postman-Caucheteux et al., Single-trial fMRI shows contralesional activity linked to overt naming errors in chronic aphasic patients. J. Cogn. Neurosci. 22, 1299-1318 (2010).

50. D. Saur et al., Dynamics of language reorganization after stroke. Brain 129, 1371-1384 (2006)

51. J. P. Szaflarski, J. B. Allendorfer, C. Banks, J. Vannest, S. K. Holland, Recovered vs. notrecovered from post-stroke aphasia: The contributions from the dominant and non dominant hemispheres. Restor. Neurol. Neurosci. 31, 347-360 (2013).

52. J. P. Szaflarski et al., Poststroke aphasia recovery assessed with functional magnetic resonance imaging and a picture identification task. J. Stroke Cerebrovasc. Dis. 20 336-345 (2011)

53. C. A. van Oers et al., Contribution of the left and right inferior frontal gyrus in re covery from aphasia. A functional MRI study in stroke patients with preserved hemodynamic responsiveness. Neuroimage 49, 885-893 (2010)

54. E. Warburton, C. J. Price, K. Swinburn, R. J. S. Wise, Mechanisms of recovery from aphasia: Evidence from positron emission tomography studies. J. Neurol. Neurosurg. Psychiatry 66, 155-161 (1999).
55. S. C. Blank, H. Bird, F. Turkheimer, R. J. Wise, Speech production after stroke: The role of the right pars opercularis. Ann. Neurol. 54, 310-320 (2003)

56. A. Basso, M. Gardelli, M. P. Grassi, M. Mariotti, The role of the right hemisphere in recovery from aphasia. Two case studies. Cortex 25, 555-566 (1989)

57. P. E. Turkeltaub, Brain stimulation and the role of the right hemisphere in aphasia recovery. Curr. Neurol. Neurosci. Rep. 15, 72 (2015).

58. P. I. Martin et al., Transcranial magnetic stimulation as a complementary treatment for aphasia. Semin. Speech Lang. 25, 181-191 (2004).

59. M. A. Naeser et al., Improved picture naming in chronic aphasia after TMS to part of right Broca's area: An open-protocol study. Brain Lang. 93, 95-105 (2005).

60. P. E. Turkeltaub et al., The right hemisphere is not unitary in its role in aphasia re covery. Cortex 48, 1179-1186 (2012).

61. L. Winhuisen et al., Role of the contralateral inferior frontal gyrus in recovery of language function in poststroke aphasia: A combined repetitive transcranial magnetic stimulation and positron emission tomography study. Stroke 36, 1759-1763 (2005).

62. C. Calautti, F. Leroy, J. Y. Guincestre, J. C. Baron, Dynamics of motor network overactivation after striatocapsular stroke: A longitudinal PET study using a fixedperformance paradigm. Stroke 32, 2534-2542 (2001).

63. R. S. Marshall et al., Evolution of cortical activation during recovery from corticospinal tract infarction. Stroke 31, 656-661 (2000)

64. N. S. Ward, M. M. Brown, A. J. Thompson, R. S. Frackowiak, Neural correlates of motor recovery after stroke: A longitudinal fMRI study. Brain 126, 2476-2496 (2003).

65. R. Chu, J. A. Meltzer, T. Bitan, Interhemispheric interactions during sentence com prehension in patients with aphasia. Cortex 109, 74-91 (2018)

66. T. M. Schofield et al., Changes in auditory feedback connections determine the severity of speech processing deficits after stroke. J. Neurosci. 32, 4260-4270 (2012).

67. E. Bates et al., Voxel-based lesion-symptom mapping. Nat. Neurosci. 6, 448-450 (2003).

68. R. A. Butler, M. A. Lambon Ralph, A. M. Woollams, Capturing multidimensionality in stroke aphasia: Mapping principal behavioural components to neural structures. Brain 137, 3248-3266 (2014)

69. L. K. Tyler, W. Marslen-Wilson, E. A. Stamatakis, Dissociating neuro-cognitive component processes: Voxel-based correlational methodology. Neuropsychologia 43, 771-778 (2005).

70. D. Saur et al., Early functional magnetic resonance imaging activations predict language outcome after stroke. Brain 133, 1252-1264 (2010)

71. S. Fischer-Baum, A. Jang, D. Kajander, The cognitive neuroplasticity of reading recovery following chronic stroke: A representational similarity analysis approach. Neural Plast. 2017, 2761913 (2017)

72. K. Dilkina, J. L. McClelland, D. C. Plaut, A single-system account of semantic and lexical deficits in five semantic dementia patients. Cogn. Neuropsychol. 25, 136-164 (2008).

73. P. Belin et al., Recovery from nonfluent aphasia after melodic intonation therapy: A PET study. Neurology 47, 1504-1511 (1996).

74. Y.-N. Chang, S. Welbourne, C.-Y. Lee, Exploring orthographic neighborhood size effects in a computational model of Chinese character naming. Cognit. Psychol. 91, 1-23 (2016).

75. L. Chen, M. A. Lambon Ralph, T. T. Rogers, A unified model of human semantic knowledge and its disorders. Nat. Hum. Behav. 1, 0039 (2017).

76. M. S. Gazzaniga, Cerebral specialization and interhemispheric communication: Does the corpus callosum enable the human condition? Brain 123, 1293-1326 (2000).

77. H. W. R. Powell et al., Hemispheric asymmetries in language-related pathways: A combined functional MRI and tractography study. Neuroimage 32, 388-399 (2006)

78. D. V. M. Bishop, Cerebral asymmetry and language development: Cause, correlate, or consequence? Science 340, 1230531 (2013).

79. A. C. Schapiro, J. L. McClelland, S. R. Welbourne, T. T. Rogers, M. A. Lambon Ralph Why bilateral damage is worse than unilateral damage to the brain. J. Cogn. Neurosci. 25, 2107-2123 (2013).

80. D. C. Plaut, "Systematicity and specialization in semantics" in Connectionist Models in Cognitive Neuroscience. Perspectives in Neural Computing, D. Heinke, G. W. Humphreys, A. Olson, Eds. (Springer, London, 1999), pp. 184-193.

81. J. Morton, Interaction of information in word recognition. Psychol. Rev. 76, 165-178 (1969)

82. C. Anglade, A. Thiel, A. I. Ansaldo, The complementary role of the cerebral hemispheres in recovery from aphasia after stroke: A critical review of literature. Brain Inj. 28, 138-145 (2014).

83. J. Andoh, T. Paus, Combining functional neuroimaging with off-line brain stimulation: Modulation of task-related activity in language areas. J. Cogn. Neurosci. 23, 349-361 (2011).

84. G. Hickok, D. Poeppel, Towards a functional neuroanatomy of speech perception. Trends Cogn. Sci. 4, 131-138 (2000)

85. G. Hickok, D. Poeppel, The cortical organization of speech processing. Nat. Rev. Neurosci. 8, 393-402 (2007)

86. J. P. Rauschecker, S. K. Scott, Maps and streams in the auditory cortex: Nonhuma primates illuminate human speech processing. Nat. Neurosci. 12, 718-724 (2009).

87. D. Saur et al., Ventral and dorsal pathways for language. Proc. Natl. Acad. Sci. U.S.A $105,18035-18040$ (2008)

88. N. Kriegeskorte, Deep neural networks: A new framework for modeling biological vision and brain information processing. Annu. Rev. Vis. Sci. 1, 417-446 (2015).

89. T. P. Lillicrap, A. Santoro, L. Marris, C. J. Akerman, G. Hinton, Backpropagation and the brain. Nat. Rev. Neurosci. 21, 335-346 (2020).

90. J. L. Elman, Finding structure in time. Cogn. Sci. 14, 179-211 (1990).

91. Y.-N. Chang, P. Monaghan, S. Welbourne, A computational model of reading across development: Effects of literacy onset on language processing. J. Mem. Lang. 108, 104025 (2019).

92. M. W. Harm, M. S. Seidenberg, Computing the meanings of words in reading: Co operative division of labor between visual and phonological processes. Psychol. Rev. $111,662-720$ (2004)

93. M. Marcus, B. Santorini, M. A. Marcinkiewicz, Building a large annotated corpus of English: The Penn Treebank. Comput. Linguistics 19, 313-330 (1993).

94. N. Kriegeskorte, M. Mur, P. Bandettini, Representational similarity analysis-Connecting the branches of systems neuroscience. Front. Syst. Neurosci. 2, 4 (2008). 